\title{
THE VALUATIVE TREE OF A TWO-DIMENSIONAL REGULAR LOCAL RING
}

\author{
A. Granja
}

\begin{abstract}
We prove that the set of normalized centered real valuations of a twodimensional regular noetherian local ring has a natural structure of parameterized, rooted, non-metric tree performed on the field of real numbers.
\end{abstract}

\section{Introduction}

Throughout this paper all the rings considered will be commutative and with unit element. For a local ring $R$, we denote by $M(R)$ its maximal ideal and by $\operatorname{dim}(R)$ the Krull dimension of $R$.

Let $R$ be a noetherian local ring and let us denote by $\mathcal{V}$ the valuation space attached to $R$, whose elements are functions $\nu: R \longrightarrow \overline{\mathbb{R}}_{+}=[0, \infty]$ satisfying the standard axioms of valuations and normalized by $\nu(M(R))=\min \{\nu(y) ; y \in M(R)\}=1$.

In [FJ1], Favre and Jonsson have shown that $(\mathcal{V}, \leq)$ has the structure of a parameterized, rooted, non-metric tree, when $R=\mathbb{C}[[X, Y]]$ is the power series ring in two variables $X, Y$ over the complex field $\mathbb{C}$ and $\mu \leq \nu$ iff $\mu(f) \leq \nu(f)$ for all $f \in R$ and $\mu, \nu \in \mathcal{V}$. The tree structure of $\mathcal{V}$ provides an efficient means of encoding singularities given in terms of measures on $\mathcal{V}$. The construction of these measures uses a natural tree Laplace operator and is developed for a general rooted non-metric tree, with an increasing parameterization. This is used in [FJ1], [FJ2] and [FJ3] to study curves, ideals and plurisubharmonic functions in a unified way.

The main tool in [FJ1] is to associate a sequence of key polynomials (SKP) for each valuation $\nu \in \mathcal{V}$, which determines and is determined by $\nu$. This is nothing but the adaptation of MacLane's method of [M1] and [M2]. Furthermore, among several others, relations between $\mathcal{V}$ and Puiseux series or dual graph are also set up.

The possible extension of the above results to more general rings is discussed in Appendix E of [FJ1]. In particular, it would be interesting to extend some of them when either $R=K[[X, Y]]$ is the power series ring in two variables over a field $K$ (for instance of positive characteristic or not algebraically closed) or, more generally, when $R$ is a two-dimensional regular noetherian local ring (perhaps non-equicharacteristic). In these cases, SKP or Puiseux series fail and we need a different approach.

The objective of this paper is to show that $(\mathcal{V}, \leq)$ is a parameterized, rooted, non-metric tree, when $R$ is a two-dimensional regular noetherian local ring.

For this, we consider the sequence of quadratic dilatations $\left\{\left(R_{i}^{\nu}, \nu^{i}\right)\right\}_{i=0}^{\lambda(\nu)}$ (with $\lambda(\nu)$ possibly infinite) of $R$ along $\nu \in \mathcal{V}$. Here $\nu^{i}$ is a valuation on $R_{i}^{\nu}$, whose restriction to $R=R_{0}^{\nu}$ is $\nu$. Also and as in [GR] or [L], we consider the multiplicity

Received by the editors October 24, 2005.

Research partially supported by DGICYT, MTM 2005-05207 and JCYL, LE026A06. 
sequence $\left\{m_{i}^{\nu}\right\}_{i=0}^{\lambda(\nu)}$ attached to the pair $(R, \nu)$, where $m_{i}^{\nu}=\min \left\{\nu^{i}(y) ; y \in M\left(R_{i}^{\nu}\right)\right\}$ for $0 \leq i<\lambda(\nu)$ and $m_{\lambda(\nu)}^{\nu}=0$, in the case where $\lambda(\nu)<\infty$. The sequence of quadratic dilatations determines and is determined by $\nu$, in fact, $\bigcup_{i=0}^{\lambda(\nu)} R_{i}^{\nu}=V$ is a valuation ring. Notice that if $\nu^{-1}(\infty) \neq\{0\}$, then $\nu$ is not the valuation associated with $V$ and this corresponds with the case of a curve valuation (defined by the prime principal ideal $\left.\nu^{-1}(\infty)\right)$ on the sense of [FJ1], (1.5.5).

There is a kind of valuations of $\mathcal{V}$ that play a central role in [FJ1] as well as in our study: quasimonomial valuations. A valuation $\nu$ is quasimonomial if $\nu^{i}$ is a monomial valuation for some $i \geq 0$. Monomial valuations are nothing but the valuations induced by a usual monomial valuation on the graduate ring $G_{M(R)}(R)$. Quasimonomial valuations have two main properties. First, $\nu$ is quasimonomial if and only if the set $\mathcal{F}(\nu)=\left\{i ; R_{i}^{\nu}\right.$ is free $\}$ is finite. Here the concept of free or satellite for some $R_{i}^{\nu}$ cames from the proximity relations on the sequence of quadratic dilatations. (See, for example, [GR] or [L]). Second and as consequence of our Theorem 18, if $\mu<\nu$, then $\mu$ is quasimonomial.

With this background, we can define $\mathcal{G}(\nu)=\sum_{i \in \mathcal{F}(\nu)} m_{i}^{\nu}$ for each $\nu \in \mathcal{V}$, that has the good properties that allows us to show that $(\mathcal{V}, \leq)$ is a parameterized (by $\mathcal{G}$ ), rooted, non-metric tree.

A part from this Introduction, the paper is organized into four further sections that we will describe next. The first section is technical in nature and contains concepts and properties about valuations, quadratic dilatations, multiplicity sequence and proximity relations. Section 3 focuses on the concept and properties of quasimonomial valuations. In Section 4, we study the relations between the above sequences associated with two centered valuations $\mu$ and $\nu$ on $R$, provided that $\mu(f) \leq \nu(f)$ for all $f \in R$. The last section is devoted to the proof of our main result, Theorem 29, which is obtained from several previous results that state the properties of $\mathcal{G}$.

In what follows, $R$ will be a regular noetherian local ring of dimension two and we will denote by $\operatorname{Ord}_{R}(f)$ the usual multiplicity of $f \in R-\{0\}$, i.e. the non-negative integer $d$ such that $f \in(M(R))^{d}$ and $f \notin(M(R))^{d+1}$.

\section{Notations and preliminaries}

This section is technical in nature and contains the initial concepts, notations and properties.

We write $\overline{\mathbb{R}}_{+}=\mathbb{R}_{+} \cup\{\infty\}=[0, \infty]$, where $\mathbb{R}_{+}$is the set of non-negative real numbers with $0 \in \mathbb{R}_{+}$and where we extend addition, multiplication and order on $\mathbb{R}_{+}$ to $\overline{\mathbb{R}}_{+}$in the usual way.

A valuation on $R$ is a non-constant function $\nu: R \longrightarrow \overline{\mathbb{R}}_{+}$such that

(V1) $\nu(x y)=\nu(x)+\nu(y)$ for all $x, y \in R$;

(V2) $\nu(x+y) \geq \min \{\nu(x), \nu(y)\}$ for all $x, y \in R$;

(V3) $\nu(1)=0$.

Note that $\nu(0)=\infty$ and $\nu(R) \geq 0$.

A valuation $\nu$ is proper if $\nu(R) \neq\{0, \infty\}$ and is centered if $\nu$ is proper and $\nu(M(R))>0$. 
Notice that $\operatorname{Ord}_{R}$ is a centered valuation on $R$ by setting $\operatorname{Ord}_{R}(0)=\infty$ and that if $\mathfrak{p}$ is a prime ideal of $R$ such that $\nu^{-1}(\infty) \subset \mathfrak{p}$, then $\nu$ extend to a valuation on $R_{\mathfrak{p}}$ in the obvious form.

The above concept of valuation is a restricted version of the more general concept of Manis valuation when one take a totally ordered abelian group $\Gamma$ instead of the additive group of real numbers $\mathbb{R}$, i. e. Manis valuations are non-constant maps $\nu: R \longrightarrow \Gamma \cup\{\infty\}$ satisfying (V1)-(V3). Furthermore, we obtain the usual concept of valuation or Krull valuation when $\nu^{-1}(\infty)=\{0\}$. In this case, $\nu$ extends to a Krull valuation (also denoted by $\nu$ ) on the quotient field $K(R)$ of $R$, $\nu(K(R)-\{0\})$ is a totally ordered subgroup of $\Gamma$ (called the group of values of $\nu$ ) and $V_{\nu}=\{x \in K(R) ; \nu(x) \geq 0\}$ is the valuation ring associated to $\nu$, which is a local ring with maximal ideal $M\left(V_{\nu}\right)=\{x \in K(R) ; \nu(x)>0\}$. In addition, let us remember that the rank of a Krull valuation $\nu$ (denoted rank $(\nu)$ ) is the Krull dimension of the ring $V_{\nu}$ and is also the least integer $l$ such that $\nu(K(R)-\{0\})$ can be embedded as an ordered group into $\left(\mathbb{R}^{l},+\right)$ endowed with the lexicographic order. In our case, as $\operatorname{dim}(R)=2$, we have rank $(\nu) \leq 2$ by Abhyankar's inequalities, see, for example, [ZS].

In this form, centered valuations $\nu$ on $R$ with $\nu^{-1}(\infty)=\{0\}$ are nothing but real rank one centered Krull valuations on $R$. Furthermore, if $\nu$ is a centered valuation on $R$ with $\nu^{-1}(\infty) \neq\{0\}$, then $\nu$ determines a Krull valuation on $R$ of real rank two as follows: $\nu^{-1}(\infty)$ is a principal prime ideal generated by an irreducible element $f \in R$ and we can define $\omega: R-\{0\} \longrightarrow(\mathbb{Z} \oplus \mathbb{R})$ by $\omega(g)=\left(s, \nu\left(g^{\prime}\right)\right)$ for each $g \in R-\{0\}$, where $g=g^{\prime} f^{s}, g^{\prime} \notin f R$ and $\mathbb{Z} \oplus \mathbb{R}$ is lexicographically ordered. In particular, with each centered valuation $\nu$ on $R$ it is possible to associate a unique valuation ring $V$ of $K(R)$ that birationally contains $R$ and such that its associated Krull valuation has real rank one. Namely, if $\nu^{-1}(\infty)=\{0\}$, then $\nu$ is a real rank one centered Krull valuation and $V=V_{\nu}$ is its valuation ring; and if $\nu^{-1}(\infty) \neq\{0\}$, then $V=R_{\nu^{-1}(\infty)}$ is a discrete valuation ring such that $V_{\omega} \subsetneq V=R_{\nu^{-1}(\infty)}$. Notice that in the last case if $R / \nu^{-1}(\infty)$ is an analytically irreducible ring (for example, if $R$ is a complete or henselian ring), then the valuation ring $V_{\omega}$ (of real rank two) is univocally determined by the height one prime ideal $\nu^{-1}(\infty)$.

The converse is also true, every real rank two centered Krull valuation $\omega$ such that $\mathfrak{p} \cap R \neq M(R)$ determines a centered valuation $\nu$ on $R$ as follows: $\nu(g)=p r_{2}(\omega(g))$ when $g \notin \mathfrak{p} \cap R$ and $\nu(g)=\infty$ when $g \in \mathfrak{p} \cap R$. Here $0 \subset \mathfrak{p} \subset M(W)$ are the prime ideals of the valuation ring $W$ of $\omega$ and $p r_{2}: \mathbb{R} \oplus \mathbb{R} \longrightarrow \mathbb{R}$ is the second projection. The remainder centered Krull valuations of real rank two with $\mathfrak{p} \cap R=M(R)$ define the non-proper valuation $\nu$ with $\nu(M(R))=\infty$ and $\nu(R-M(R))=0$.

Finally, we point out that each centered valuation (or Krull valuation) $\nu$ on $R$ extends to a unique valuation $\hat{\nu}$ on the $M(R)$-adic completion $\hat{R}$ of $R$. Note that, in general, one can have $\nu^{-1}(\infty)=\{0\}$ and $\hat{\nu}^{-1}(\infty) \neq\{0\}$ (or in terms of Krull valuations $1=\operatorname{rank}(\nu)<\operatorname{rank}(\hat{\nu})=2)$.

Next, we recall the concepts of quadratic dilatation and multiplicity sequence.

A quadratic dilatation of $R$ is a ring $R_{1}=\left(R\left[z^{-1} M(R)\right]\right)_{\mathfrak{q}}$, where $z \in M(R)-(M(R))^{2}$ and $\mathfrak{q}$ is a prime ideal of $R\left[z^{-1} M(R)\right]$ such that $M(R) R\left[z^{-1} M(R)\right] \subset \mathfrak{q}$.

For the rest of this section, let us fix a centered valuation $\nu$ on $R$. 
Let $\left(x_{1}, x_{2}\right)$ be a base of $M(R)$ such that $0<\nu\left(x_{1}\right) \leq \nu\left(x_{2}\right)$ and let us write $A=R\left[\frac{x_{2}}{x_{1}}\right]=R\left[\left(x_{1}\right)^{-1} M(R)\right]$ and $\nu^{1}: A \longrightarrow \overline{\mathbb{R}}_{+}$the unique extension of $\nu$ to $A$ such that $\nu^{1}\left(x_{2} / x_{1}\right)=\nu\left(x_{2}\right)-\nu\left(x_{1}\right)$. We have $\nu^{1}(A) \geq 0, M(R) A=x_{1} A \subset Q=$ $\left\{y \in A ; \nu^{1}(y)>0\right\}$ and $R_{1}^{\nu}=A_{Q}$ is a quadratic dilatation of $R$, that is called the quadratic dilatation of $R$ along $\nu$. Moreover, $R_{1}^{\nu}$ is a regular noetherian local ring with $1 \leq \operatorname{dim}\left(R_{1}^{\nu}\right) \leq \operatorname{dim}(R)=2$ and $R_{1}^{\nu}$ is univocally determined by $\nu$.

Notice that if $\operatorname{dim}\left(R_{1}^{\nu}\right)=1$, then $Q=M(R) A=x_{1} A$ and $R_{1}^{\nu}=A_{M(R) A}=A_{x_{1} A}$ is the (discrete) valuation ring associated to $\operatorname{Ord}_{R}$. (In fact, $\nu=a \cdot \operatorname{Ord}_{R}$ for some $a \in \mathbb{R}, a>0$ ). If $\operatorname{dim}\left(R_{1}^{\nu}\right)=2$, then $\nu$ extends to a centered valuation (also denoted $\nu^{1}$ ) on $R_{1}^{\nu}$ and there exists the quadratic dilatation $R_{2}^{\nu}$ of $R_{1}^{\nu}$ along $\nu^{1}$. Thus we have a (possibly infinite) sequence $R=R_{0}^{\nu} \subset R_{1}^{\nu} \subset \cdots \subset R_{i}^{\nu} \subset \cdots$, such that $R_{i}^{\nu}$ is the quadratic dilatation of $R_{i-1}^{\nu}$ along $\nu^{i-1}, i \geq 1$. Here $\nu=\nu^{0}$.

We denote by $\lambda(\nu)$ the length of the above sequence. Note that $\operatorname{dim}\left(R_{j}\right)=1$ for some $j \geq 0$ iff $\lambda(\nu)<\infty$, and, in this case, $\nu=a \cdot \operatorname{Ord}_{R_{\lambda(\nu)-1}}$ for some $a \in \mathbb{R}, a>0$. Furthermore, $\nu$ extends to a centered valuation $\nu^{i}$ on $R_{i}^{\nu}$ such that $\nu^{-1}(\infty) \neq(0)$ iff $\left(\nu^{i}\right)^{-1}(\infty) \neq(0), 0 \leq i \leq \lambda(\nu)$. In particular, $\nu$ is a Krull valuation iff $\nu^{i}$ is a Krull valuation for $i \geq 0$.

The sequence $\left\{\left(R_{i}^{\nu}, \nu^{i}\right)\right\}_{i=0}^{\lambda(\nu)}$ is called the sequence of quadratic dilatations of $R$ along $\nu$. We note that $\bigcup_{i=0}^{\lambda(\nu)} R_{i}^{\nu}=V_{\nu}$ is a valuation ring, (see [Ab]) that is called the valuation ring associated with $\nu$. In fact, $V_{\nu}$ is the valuation ring of the Krull valuation $\omega$ defined by $\nu$ as above.

As in $[\mathrm{GR}]$ or $[\mathrm{L}]$, we attach to the pair $(R, \nu)$ the multiplicity sequence $\left\{m_{i}^{\nu}\right\}_{i=0}^{\lambda(\nu)}$ given by $m_{i}^{\nu}=\min \left\{\nu_{i}(y) ; y \in M\left(R_{i}^{\nu}\right)\right\}$, for $0 \leq i<\lambda(\nu)$ and by $m_{\lambda(\nu)}^{\nu}=0$, if $\lambda(\nu)<\infty$.

On the other hand, let us consider $g \in R-\{0\}$. The strict transform of $(R, g R)$ in $R_{1}^{\nu}$ is the pair $\left(R_{1}^{\nu}, g_{1} R_{1}^{\nu}\right)$ such that $g_{1} R_{1}^{\nu}$ is the unique principal ideal given by $(M(R))^{d} g_{1} R_{1}^{\nu}=g R_{1}^{\nu}$, where $d=\operatorname{Ord}_{R^{\nu}}(g)$.

In general, for $0 \leq j \leq \lambda(\nu)$ the strict transform of $(R, g R)$ in $R_{j}^{\nu}$ is the pair $\left(R_{j}^{\nu}, g_{j} R_{j}^{\nu}\right)$ defined inductively as follows: If $j=0$, then $(R, g R)=\left(R_{j}^{\nu}, g_{j} R_{j}^{\nu}\right)$; and if $j>i$ and $\left(R_{j-1}^{\nu}, g_{j-1} R_{j-1}^{\nu}\right)$ is the strict transform of $(R, g R)$ in $R_{j-1}^{\nu}$, then $\left(R_{j}^{\nu}, g_{j} R_{j}^{\nu}\right)$ is the strict transform of $\left(R_{j-1}^{\nu}, g_{j-1} R_{j-1}^{\nu}\right)$ in $R_{j}^{\nu}$.

We point out that if $\lambda(\nu)<\infty$, then $g_{\lambda(\nu)} R_{\lambda(\nu)}^{\nu}=R_{\lambda(\nu)}^{\nu}$.

Lemma 1. With the above notations, we have the following statements:

1) If $\lambda(\nu)=\infty$ and $g_{j} R_{j}^{\nu} \neq R_{j}^{\nu}$ for all $j \geq 0$, then there exists $i_{0} \geq 0$ such that $\operatorname{Ord}_{R_{j}^{\nu}}\left(g_{j}\right)=\operatorname{Ord}_{R_{j+1}^{\nu}}\left(g_{j+1}\right)$ and $m_{j}^{\nu}=m_{j+1}^{\nu}$, for $j \geq i_{0}$.

2) If $g \notin(\nu)^{-1}(\infty)$, then there exists $i_{0}(g) \geq 0$ such that $g_{j} R_{j}^{\nu}=R_{j}^{\nu}$, for $j \geq i_{0}(g)$. Moreover, $\nu(g)=\sum_{j \geq 0} m_{j}^{\nu} \cdot \operatorname{Ord}_{R_{j}^{\nu}}\left(g_{j}\right)$.

Proof. 1) We note that $\operatorname{Ord}_{R_{j}^{\nu}}\left(g_{j}\right) \geq \operatorname{Ord}_{R_{j+1}^{\nu}}\left(g_{j+1}\right)$ and $m_{j+1}^{\nu} \geq m_{j}^{\nu}$ for $j \geq 0$. Thus, there exists $i_{0} \geq 0$ with $\operatorname{Ord}_{R_{j}^{\nu}}\left(g_{j}\right)=\operatorname{Ord}_{R_{j+1}^{\nu}}\left(g_{j+1}\right)$ for $j \geq i_{0}$.

Let us consider $j \geq i_{0}$ and a base $(x, y)$ of $M\left(R_{j}^{\nu}\right)$ with $\nu^{j}(x)=m_{j}^{\nu} \leq \nu^{j}(y)$. We can write $g_{j}=\sum_{k+l=d} a_{k l} x^{k} y^{l}+g_{j}^{\prime}$, with $d=\operatorname{Ord}_{R_{j}^{\nu}}\left(g_{j}\right), g_{j}^{\prime} \in\left(M\left(R_{j}^{\nu}\right)\right)^{d+1}$ and either $a_{k l}=0$ or $a_{k l} \notin M\left(R_{j}^{\nu}\right)$ when $k+l=d$. Therefore, $g_{j+1}=\sum_{k+l=d} a_{k l}(y / x)^{l}+x h_{j+1}$, with $h_{j+1} \in R_{j+1}^{\nu}$. 
Since $\operatorname{Ord}_{R_{j+1}^{\nu}}\left(g_{j+1}\right)=d=\operatorname{Ord}_{R_{j}^{\nu}}\left(g_{j}\right)$, there exists $\alpha, a \in R_{j}^{\nu}$ with $a \notin M\left(R_{j}^{\nu}\right)$ and either $\alpha=0$ or $\alpha \notin M\left(R_{j}^{\nu}\right)$ such that $\sum_{k+l=d} a_{k l} x^{k} y^{l}-a(y+\alpha x)^{d} \in\left(M\left(R_{j}^{\nu}\right)\right)^{d+1}$. Thus, and after a suitable change of the base, we can assume $\alpha=0$ and write $g_{j+1}=a(y / x)^{d}+x h_{j+1}$, with $h_{j+1} \in R_{j+1}^{\nu}$.

Now, if $\nu^{j+1}(y / x)<\nu^{j+1}(x)=\nu^{j}(x)=m_{j}^{\nu}$, then $y / x \notin M\left(R_{j+1}^{\nu}\right)$. So $g_{j+2} R_{j+2}^{\nu}=R_{j+2}^{\nu}$ and we reach a contradiction. Hence, $\nu^{j+1}(y / x) \geq \nu^{j+1}(x)=$ $\nu^{j}(x)=m_{j}^{\nu}$ and $m_{j+1}^{\nu}=m_{j}^{\nu}$.

2) For $0 \leq j \leq \lambda(\nu)$, we have $\nu^{j}\left(g_{j}\right)=\nu^{j+1}\left(g_{j}\right)=\nu^{j+1}\left(x^{d} g_{j+1}\right)=d m_{j}^{\nu}+$ $\nu^{j+1}\left(g_{j+1}\right)$, where $d=\operatorname{Ord}_{R_{j}^{\nu}}\left(g_{j}\right), x R_{j+1}^{\nu}=M\left(R_{j}^{\nu}\right) R_{j+1}^{\nu}$ and $\nu^{j}(x)=m_{j}^{\nu}$.

If $\lambda(\nu)<\infty$, then $g_{\lambda(\nu)} R_{\lambda(\nu)}^{\nu}=R_{\lambda(\nu)}^{\nu}$ and there exists $i_{0}(g) \geq 0$ such that $g_{j} R_{j}^{\nu}=$ $R_{j}^{\nu}$, for $i_{0}(g) \leq j \leq \lambda(\nu)$.

If $\lambda(\nu)=\infty$ and $g_{j} R_{j}^{\nu} \neq R_{j}^{\nu}$ for $j \geq 0$, then, by 1$)$, there exists $i_{1} \geq 0$ such that $\operatorname{Ord}_{R_{j}^{\nu}}\left(g_{j}\right)=\operatorname{Ord}_{R_{j+1}^{\nu}}\left(g_{j+1}\right)=d$ and $m_{j}^{\nu}=m_{j+1}^{\nu}$ for $j \geq i_{1}$. Thus, $\nu^{i_{1}}\left(g_{i_{1}}\right) \geq$ $\sum_{j=i_{1}}^{k} d m_{j}^{\nu}$, for all $k \geq i_{1}$ and $\nu^{i_{1}}\left(g_{i_{1}}\right)=\infty$. So, as $\nu^{j}\left(g_{j}\right) \geq \nu^{j+1}\left(g_{j+1}\right)$ for $j \geq 0$, we have $\nu(g)=\infty$, which is a contradiction. Hence, there exists $i_{0}(g) \geq 0$ such that $g_{j} R_{j}^{\nu}=R_{j}^{\nu}$, for $j \geq i_{0}(g)$.

Finally, as $\nu^{j}\left(g_{j}\right)=0$ for $j \geq i_{0}(g)$ and $\nu^{j}\left(g_{j}\right)=\operatorname{Ord}_{R_{j}^{\nu}}\left(g_{j}\right) \cdot m_{j}^{\nu}+\nu^{j+1}\left(g_{j+1}\right)$ for $j \geq 0$, then $\nu(g)=\sum_{j \geq i} m_{j}^{\nu} \operatorname{Ord}_{R_{j}}\left(g_{j}\right)$.

To finish this section we will outline some concepts and results concerning proximity relations.

For $0 \leq i<j \leq \lambda(\nu)$, we say that $R_{j}^{\nu}$ is proximate to $R_{i}^{\nu}$ if the valuation ring of $\operatorname{Ord}_{R_{i}^{\nu}}$ contains $R_{j}^{\nu}$. In what follows, $\mathcal{P}\left(R_{i}^{\nu}\right)=\left\{R_{j}^{\nu} ; R_{j}^{\nu}\right.$ is proximate to $\left.R_{i}^{\nu}\right\}$ denotes the set of proximate points of $R_{i}^{\nu}$, for $i \geq 0$ and we note that $R_{j}^{\nu}$ is proximate to $R_{j-1}^{\nu}$ and at most to one other ring. (See $[\mathrm{L}]$ ).

We say that $R_{j}^{\nu}$ is free (or a free point) if either $j=0$ or $j \geq 1$ and $R_{j}^{\nu}$ is proximate to one ring only, namely $R_{j-1}$. Otherwise, we say that $R_{j}^{\nu}$ is satellite (or a satellite point). Note that $R=R_{0}^{\nu}$ and $R_{1}^{\nu}$ are free and also if $\lambda(\nu)<\infty$, then $R_{\lambda(\nu)}^{\nu}$ is free. We will denote by $\mathcal{F}(\nu)=\left\{i ; R_{i}^{\nu}\right.$ is free $\}$.

Remark 2. With the above notations, let us assume $0 \leq i<i+2 \leq \lambda(\nu)$, then $m_{i+1}^{\nu}=m_{i}^{\nu}$ iff $R_{i+2}^{\nu} \notin \mathcal{P}\left(R_{i}^{\nu}\right)$. (See Lemma 16 of [GR]). Moreover, if $\mathcal{P}\left(R_{i}^{\nu}\right)$ is a finite set, then $m_{i}^{\nu}=\sum_{R_{j}^{\nu} \in \mathcal{P}\left(R_{i}^{\nu}\right)} m_{j}^{\nu}$. (See Lemma 17 of [GR]).

Lemma 3. With the above notations, $\mathcal{P}\left(R_{i}^{\nu}\right)$ is a finite set, for $0 \leq i<\lambda(\nu)$.

Proof. If $\mathcal{P}\left(R_{i}^{\nu}\right)$ is not finite, then $\lambda(\nu)=\infty$ and for each $j>i$ we can take a base $\left(x_{1}^{(j)}, x_{2}^{(j)}\right)$ of $M\left(R_{j}^{\nu}\right)$ such that $M\left(R_{i}^{\nu}\right) R_{i+1}^{\nu}=x_{2}^{(i+1)} R_{i+1}$ and $M\left(R_{i}^{\nu}\right) R_{j}^{\nu}=$ $\left(x_{1}^{(j)}\right)^{a_{i j}} x_{2}^{(j)} R_{j}^{\nu}$ with $a_{i j}=j-i-1, j \geq i+1$. In fact, $x_{2}^{(j)}=x_{2}^{(i+1)} /\left(x_{1}^{(i+1)}\right)^{j-i-1}$ for $j \geq i+1$. Thus, $\nu^{i+1}\left(x_{2}^{(i+1)}\right) \geq(j-i-1) \nu^{i+1}\left(x_{1}^{(i+1)}\right)$ and $\nu^{i+1}\left(x_{2}^{(i+1)}\right)=\infty$.

As $M\left(R_{i}\right) R_{i+1}^{\nu}=x_{2}^{(i+1)} R_{i+1}^{\nu}$, then $\nu^{i}(g)=\infty$ for all $g \in M\left(R_{i}^{\nu}\right)$. In particular, $\nu(g)=\nu^{i}(g)=\infty$ for all $g \in M(R)$ and $\nu$ is not centered, which is a contradiction. Hence, $\mathcal{P}\left(R_{i}^{\nu}\right)$ is a finite set. 
Remark 4. Note that if $\mathcal{P}\left(R_{i}^{\nu}\right)=\left\{R_{i+1}^{\nu}, \ldots, R_{i+h(i)}^{\nu}\right\}$, with $h(i)>1$, then $m_{i}^{\nu}>m_{i+1}^{\nu}$ by Remark 2. Moreover, we have the following possibilities:

1) $m_{i+1}^{\nu}=\cdots=m_{i+h(i)}^{\nu}, m_{i}^{\nu}=h(i) m_{i+1}^{\nu}, R_{i+j}$ is satellite for $2 \leq j \leq h(i)$ and $R_{i+h(i)+1}$ is free.

2) $m_{i+1}^{\nu}=\cdots=m_{i+h(i)-1}^{\nu}>m_{i+h(i)}^{\nu}>0, m_{i}^{\nu}=(h(i)-1) m_{i+1}^{\nu}+m_{i+h(i)}^{\nu}$ and $R_{i+j}$ is satellite for $2 \leq j \leq h(i)+1$.

Lemma 5. With the above notations, let $(x, y)$ be a base of $M(R)$ with $\nu(x) \leq \nu(y)<$ $\infty$, then we have the following statements:

a) If $\nu(x)$ and $\nu(y)$ are rationally dependent, then there exist a non-negative integer $k \geq 1$, a sequence of non-negative integers $\left\{h_{j}\right\}_{0 \leq j \leq k}$ and a sequence of non-negative real numbers $\left\{\alpha_{j}\right\}_{0 \leq j \leq k+1}$ such that $\alpha_{0}=\nu(y), \alpha_{1}=\nu(x)$, $\alpha_{j-1}=h_{j} \alpha_{j}+\alpha_{j+1}, 1 \leq j \leq k, 0<\alpha_{j+1}<\alpha_{j}, 1 \leq j \leq k-2$ and $\alpha_{k+1}=0$. Moreover, $R_{j}^{\nu}$ is free for $0 \leq j \leq h_{1}, R_{j}^{\nu}$ is not free for $h_{1}<j<\beta$ and $R_{\beta}^{\nu}$ is free, where $\beta=h_{1}+h_{2}+\cdots+h_{k}$.

b) If $\nu(x)$ and $\nu(y)$ are rationally independent, then there exist a sequence of non-negative integers $\left\{h_{j}\right\}_{j \geq 0}$ and a sequence of non-negative real numbers $\left\{\alpha_{j}\right\}_{j \geq 0}$ such that $\alpha_{0}=\nu(y), \alpha_{1}=\nu(x), \alpha_{j-1}=h_{j} \alpha_{j}+\alpha_{j+1}$ and $0<\alpha_{j+1}<\alpha_{j}, j \geq 0$. Moreover, $R_{j}^{\nu}$ is free for $0 \leq j \leq h_{1}$ and $R_{j}^{\nu}$ is not free for $j>h_{1}$.

Proof. From the Euclidean division algorithm, we obtain either $k \geq 1,\left\{h_{j}\right\}_{0 \leq j \leq k}$ and $\left\{\alpha_{j}\right\}_{0 \leq j \leq k+1}$ in case a) or $\left\{h_{j}\right\}_{j \geq 0}$ and $\left\{\alpha_{j}\right\}_{j \geq 0}$ in case b).

Finally, the result follows from Remark 4. Note that if $h_{1}+\cdots+h_{l-1} \leq j<$ $h_{1}+\cdots+h_{l}$, then $m_{j}^{\nu}=\alpha_{l-1}$. Here $l \leq k$ in case a) and $h_{0}=0$ if $l=1$.

\section{Quasimonomial valuations}

In this section, we study the quasimonomial valuations that will play a central role in what follows. For this, we must introduce the concept of monomial valuation, which needs some previous results.

Let us fix $\left(x_{1}, x_{2}\right)$ a base of $M(R)$. We begin with the following:

Remark 6. Let $M_{1}, \ldots, M_{l+1}$ be $l+1$ pure monomials in $x_{1}$ and $x_{2}$, i.e. $M_{i}=$ $\left(x_{1}\right)^{a_{1}^{i}}\left(x_{2}\right)^{a_{2}^{i}}$, where $a_{j}^{i} \geq 0$ is a non-negative integer for $1 \leq j \leq 2$ and $1 \leq i \leq l+1$. If $M_{l+1} \in M_{1} R+\cdots+M_{l} R$, then $M_{l+1} \in M_{i} R$ for some $1 \leq i \leq l$.

Lemma 7. For each $f \in R-\{0\}$, we can write $f=a_{1} M_{1}+\cdots+a_{l} M_{l}$ such that $a_{1}, \ldots, a_{l} \notin M(R)$ and $M_{1}, \ldots, M_{l}$ are distinct pure monomials in $x_{1}$ and $x_{2}$ with $M_{i} R \not \subset M_{j} R$ for $i \neq j$. Furthermore, the monomials $M_{1}, \ldots, M_{l}$ are univocally determined by $f$ and the above conditions.

Proof. By Lemma 1.4 of $[\mathrm{Fu}]$, there exist $M_{1}, \ldots, M_{l}$ distinct pure monomials in $x_{1}$ and $x_{2}$ with $f=a_{1} M_{1}+\cdots+a_{l} M_{l}, a_{1}, \ldots, a_{l} \notin M(R)$ and $M_{i} R \not \subset M_{j} R$ for $i \neq j$. Finally, $M_{1}, \ldots, M_{l}$ are univocally determined by Remark 6 .

Let us consider $\gamma_{1}, \gamma_{2} \in \mathbb{R}_{+}$, we define $\nu: R \longrightarrow \overline{\mathbb{R}}_{+}$as follows:

- $\nu(0)=\infty$.

- $\nu\left(\left(x_{1}\right)^{\alpha_{1}}\left(x_{2}\right)^{\alpha_{2}}\right)=\alpha_{1} \gamma_{1}+\alpha_{2} \gamma_{2}$. 
- $\nu(f)=\min \left\{v\left(M_{i}\right) ; \quad 1 \leq i \leq l\right\}$, for each $f \in R-\{0\}$, where $f=a_{1} M_{1}+$ $\cdots+a_{l} M_{l}$ as in Lemma 7.

Lemma 8. With the above notations, $\nu$ is a (Krull) valuation on $R$. Moreover, if $\gamma_{i}>0$ for $1 \leq i \leq 2$, then $\nu$ is centered.

Proof. Let us consider $f, g \in R$ and write $f=\sum_{i=1}^{l} a_{i} M_{i}, g=\sum_{i=1}^{s} b_{i} M_{i}^{\prime}$, $f g=\sum_{1 \leq k \leq r} c_{k} N_{k}$ and $f+g=\sum_{1 \leq k \leq t} d_{k} N_{k}^{\prime}$ as in Lemma 7 .

By Remark 6 , we have either $N_{k}^{\prime} \in M_{i} R$ for some $1 \leq i \leq l$ or $N_{k}^{\prime} \in M_{j}^{\prime} R$ for some $1<j \leq s$. Hence, $\nu(f+g) \geq \min \{\nu(f), \nu(g)\}$.

On the other hand, since $f g=\sum_{1 \leq i \leq l, 1 \leq j \leq s} a_{i} b_{j} M_{i} M_{j}^{\prime}$, we have $\nu(f g) \geq \nu(f)+$ $\nu(g)$.

Without loss of generality, we can assume $\nu\left(M_{i}\right)=\nu(f)$ for $1 \leq i \leq l_{1}$ and $\nu\left(M_{i}\right)>\nu(f)$ for $l_{1}<i \leq l$. Similarly, $\nu\left(M_{j}^{\prime}\right)=\nu(g)$ for $1 \leq j \leq s_{1}$ and $\nu\left(M_{j}^{\prime}\right)>\nu(g)$ for $s_{1}<j \leq s$.

Let $<^{\prime}$ be the well-ordering in $\mathbb{Z}_{+}^{2}\left(\mathbb{Z}_{+}\right.$the set of non-negative integers with $0 \in \mathbb{Z}_{+}$) given by $\left(\delta_{1}, \delta_{2}\right)<^{\prime}\left(\delta_{1}^{\prime}, \delta_{2}^{\prime}\right)$, if either $\delta_{1}+\delta_{2}<\delta_{1}^{\prime}+\delta_{2}^{\prime}$ or $\delta_{1}+\delta_{2}=\delta_{1}^{\prime}+\delta_{2}^{\prime}$ and $\delta_{1}<\delta_{1}^{\prime}$ or $\delta_{1}+\delta_{2}=\delta_{1}^{\prime}+\delta_{2}^{\prime}, \delta_{1}=\delta_{1}^{\prime}$ and $\delta_{2}<\delta_{2}^{\prime}$.

Now, if $M_{i}=\left(x_{1}\right)^{\alpha_{1}^{i}}\left(x_{2}\right)^{\alpha_{2}^{i}}$ and $M_{j}^{\prime}=\left(x_{1}\right)^{\beta_{1}^{j}}\left(x_{2}\right)^{\beta_{2}^{j}}, 1 \leq i \leq l$ and $1 \leq j \leq s$, we can assume $\left(\alpha_{1}^{k}, \alpha_{2}^{k}\right)<^{\prime}\left(\alpha_{1}^{k+1}, \alpha_{2}^{k+1}\right)$ and $\left(\beta_{1}^{j}, \beta_{2}^{j}\right)<^{\prime}\left(\beta_{1}^{j+1}, \beta_{2}^{j+1}\right)$, for $1 \leq k \leq l_{1}-1$ and $1 \leq j \leq s_{1}-1$. Note that if $(i, j) \neq(1,1), 1 \leq i \leq l, 1 \leq j \leq s$, then $\nu\left(M_{1} M_{1}^{\prime}\right)<\nu\left(M_{i} M_{j}^{\prime}\right)$ or $\nu\left(M_{1} M_{1}^{\prime}\right)=\nu\left(M_{i} M_{j}^{\prime}\right)$ and $\left(\alpha_{1}^{1}+\beta_{1}^{1}, \alpha_{2}^{1}+\beta_{2}^{1}\right)<^{\prime}\left(\alpha_{1}^{i}+\right.$ $\left.\beta_{1}^{j}, \alpha_{2}^{i}+\beta_{2}^{j}\right)$. In particular, if $(i, j) \neq(1,1)$, then $M_{1} M_{1}^{\prime} \notin M_{i} M_{j}^{\prime} R$.

Since $a_{1} b_{1} \notin M(R)$, by Remark 6 , we have $M_{1} M_{1}^{\prime} \in N_{k} R$ for some $1 \leq k \leq r$. Hence, $\nu(f)+\nu(g)=\nu\left(M_{1} M_{1}^{\prime}\right) \geq \nu\left(N_{k}\right) \geq \nu(f g)$ and $\nu(f)+\nu(g)=\nu(f g)$.

Definition 9. With the above notations, we say that $\nu$ is a monomial valuation on $R$ with respect to the base $\left(x_{1}, x_{2}\right)$. In general, we say that a valuation $\nu$ on $R$ is a monomial valuation if it is monomial with respect to some base of $M(R)$.

Remark 10. We note that a monomial valuation on $R$ determines and it is determined by a monomial valuation on the graded ring of $R$. Namely, if $T_{1}$ and $T_{2}$ are indeterminates over $R / M(R)$, then Lemma 7 defines a map $\Phi$ from $R$ to the graded ring $G_{M(R)}(R)=\frac{R}{M(R)}\left[T_{1}, T_{2}\right]$ of $R$ such that $\Phi\left(x_{i}\right)=T_{i}, 1 \leq i \leq n$. Now, every centered monomial valuation $\nu$ on $R$ with respect to the base $\left(x_{1}, x_{2}\right)$ induces and it is induced by a monomial valuation $\bar{\nu}$ on $G_{M(R)}(R)$ such that $\nu\left(x_{i}\right)=\bar{\nu}\left(T_{i}\right), 1 \leq i \leq 2$.

Lemma 11. With the above notations, let $\nu$ be a valuation on $R$ such that $\nu\left(x_{1}\right)$ and $\nu\left(x_{2}\right)$ are rationally independent $\left(\nu\left(x_{1}\right) \neq \infty \neq \nu\left(x_{2}\right)\right)$. Then $\nu$ is a monomial valuation on $R$ with respect to $\left(x_{1}, x_{2}\right)$.

Proof. Straightforward, in view of Lemma 7 and Definition 9.

Before introducing quasimonomial valuations we will give a final technical result on monomial valuations.

Lemma 12. Let $\nu$ and $\mu$ be two centered valuations on $R$ such that $0 \leq \mu(f) \leq \nu(f)$ for all $f \in R$. Let us assume that $\nu$ is a monomial valuation on $R$ with respect to $\left(x_{1}, x_{2}\right)$ and that $\mu\left(x_{2}\right)=\nu\left(x_{2}\right)$. Then $\mu$ is a monomial valuation on $R$ with respect to $\left(x_{1}, x_{2}\right)$. 
Proof. Let $\mu^{\prime}$ be the monomial valuation on $R$ with respect to $\left(x_{1}, x_{2}\right)$ such that $\mu^{\prime}\left(x_{i}\right)=\mu\left(x_{i}\right)$ for $1 \leq i \leq 2$. We must see $\mu^{\prime}=\mu$.

Obviously, we have $\mu^{\prime}(g) \leq \mu(g)$ for all $g \in R$. Let us assume $\mu^{\prime} \neq \mu$, then there exists an irreducible element $f \in R-\{0\}$ such that $\mu^{\prime}(f)<\mu(f)$. Note that $f \notin x_{1} R$ (otherwise $f R=x_{1} R$ and $\left.\mu^{\prime}(f)=\mu(f)\right)$ and that $\mu\left(x_{1}\right)<\nu\left(x_{1}\right)$ (otherwise $\mu\left(x_{1}\right)=\nu\left(x_{1}\right)=\mu^{\prime}\left(x_{1}\right)$ and $\left.\mu=\nu=\mu^{\prime}\right)$.

Let us write $f=a_{1} M_{1}+\cdots+a_{l} M_{l}$ as in Lemma 7. Since $\mu^{\prime}$ is monomial, we can assume, without loss of generality, that $\mu^{\prime}\left(M_{i}\right)=\mu^{\prime}(f), 1 \leq i \leq l$. Also, as $f \notin x_{1} R$, we can assume $M_{1} \notin x_{1} R$. Hence, $\mu^{\prime}\left(M_{1}\right)=\mu\left(M_{1}\right)=\nu\left(M_{1}\right)$.

Now, if $M_{i} \in x_{1} R$, then $\mu\left(M_{i}\right)=\mu^{\prime}\left(M_{i}\right)=\mu^{\prime}(f)<\nu\left(M_{i}\right)$; and if $M_{i} \notin x_{1} R$, then $\mu\left(M_{i}\right)=\mu^{\prime}\left(M_{i}\right)=\mu^{\prime}(f)=\nu\left(M_{i}\right)$. Thus, if $M_{j} \in x_{1} R$ and $M_{i} \notin x_{1} R$, we have $\nu\left(M_{j}\right)>\mu\left(M_{j}\right)=\mu^{\prime}\left(M_{j}\right)=\mu^{\prime}(f)=\mu^{\prime}\left(M_{i}\right)=\mu\left(M_{i}\right)=\nu\left(M_{i}\right)$. Hence, $\mu^{\prime}(f)=\mu^{\prime}\left(M_{1}\right)=\nu\left(M_{1}\right)=\nu(f) \geq \mu(f)>\mu^{\prime}(f)$, which is a contradiction. Therefore, $\mu=\mu^{\prime}$.

Definition 13. Let $\nu$ be a centered valuation on $R$ and let $\left\{\left(R_{i}^{\nu}, \nu^{i}\right)\right\}_{i=0}^{\lambda(\nu)}$ be the sequence of quadratic dilatations of $R$ along $\nu$. We say that $\nu$ is a quasimonomial valuation on $R$, if $\nu^{i}$ is a monomial valuation on $R_{i}^{\nu}$ for some $i \geq 0$.

Notice that $\operatorname{Ord}_{R_{i}^{\nu}}$ is a quasimonomial valuation on $R_{j}^{\nu}$ for $0 \leq j \leq i$.

Lemma 14. With the above notations, if $\nu^{i}$ is a monomial valuation on $R_{i}^{\nu}$ for some $i<\lambda(\nu)$, then $\nu^{j}$ is a monomial valuation on $R_{j}^{\nu}$ for $i \leq j<\lambda(\nu)$.

Proof. Without loss of generality, we can assume $i=0$. Thus, $\nu$ is a monomial valuation on $R$ with respect to a base $\left(x_{1}, x_{2}\right)$ of $M(R)$ and we only need to show that $\nu^{1}$ is monomial provided that $\lambda(\nu)>1$.

Let us assume $\nu\left(x_{1}\right) \leq \nu\left(x_{2}\right)=$ and write $A=R\left[\frac{x_{2}}{x_{1}}\right]$. We have $R_{1}^{v}=A_{Q}$ and $x_{1} \in Q$, where $Q=\left\{y \in A ; \nu^{1}(y)>0\right\}$.

If $\frac{x_{2}}{x_{1}} \notin Q$, then $\nu\left(x_{1}\right)=\nu\left(x_{2}\right)$ and, as $\nu$ is monomial, then $\eta_{R_{1}^{\nu}}\left(\frac{x_{2}}{x_{1}}\right)$ is not algebraic over $\frac{R}{M(R)}$, where $\eta_{R_{1}^{\nu}}: R_{1}^{\nu} \longrightarrow \frac{R_{1}^{\nu}}{M\left(R_{1}^{\nu}\right)}$ is the canonical epimorphism. Hence, $Q A=x_{1} A$ and $\lambda(\nu)=1$. If $\frac{x_{2}}{x_{1}} \in Q$, then it is easy to check that $\nu^{1}$ is monomial on $R_{1}^{\nu}$ with respect to $\left(x_{1}, \frac{x_{2}}{x_{1}}\right)$.

Definition 15. Let $\nu$ be a centered valuation on $R$ and let $\left\{\left(R_{i}^{\nu}, \nu^{i}\right)\right\}_{i=0}^{\lambda(\nu)}$ be the sequence of quadratic dilatations of $R$ along $\nu$. The monomial height of $\nu$ is the non-negative integer or infinity given by

$$
\operatorname{mh}(\nu)=\min \left\{i \geq 0 ; \nu^{i} \text { is a monomial valuation on } R_{i}^{\nu}\right\},
$$

when $\nu$ is quasimonomial and $m h(\nu)=\infty$, otherwise.

To finish the section we characterize quasimonomial valuations in terms of proximity relations. 
Proposition 16. Let $\nu$ be a centered valuation on $R$ and $\left\{\left(R_{i}^{\nu}, \nu^{i}\right)\right\}_{i=0}^{\lambda(\nu)}$ be the sequence of quadratic dilatations of $R$ along $\nu$, then $\nu$ is a quasimonomial valuation on $R$ if and only if $\mathcal{F}(\nu)=\left\{i ; R_{i}^{\nu}\right.$ is free $\}$ is a finite set.

Proof. Let $\left\{m_{i}^{\nu}\right\}_{i=0}^{\lambda(\nu)}$ be the multiplicity sequence attached to the pair $(R, \nu)$.

First let us assume that $\nu$ is a quasimonomial, then $\nu^{j}$ is a monomial valuation on $R_{j}^{\nu}$ with respect to a base $\left(x_{1}^{(j)}, x_{2}^{(j)}\right)$ of $M\left(R_{j}^{\nu}\right)$ for some $j \geq 0$. If $\nu^{j}\left(x_{1}^{(j)}\right)$ and $\nu^{j}\left(x_{2}^{(j)}\right)$ are rationally dependent, then $\lambda(\nu)<\infty$ and $\mathcal{F}(\nu)$ is finite, by Lemma 5 , a). If $\nu^{j}\left(x_{1}^{(j)}\right)$ and $\nu^{j}\left(x_{2}^{(j)}\right)$ are rationally independent, then $\mathcal{F}\left(\nu^{j}\right)$ is finite by Lemma 5 , b). Therefore, as $\mathcal{F}(\nu) \subset\left\{R_{0}^{\nu}, R_{1}^{\nu}, \ldots R_{j-1}^{\nu}\right\} \cup \mathcal{F}\left(\nu^{j}\right)$, then $\mathcal{F}(\nu)$ is finite.

Now, let us assume that $\mathcal{F}(\nu)$ is a finite. If $\lambda(\nu)<\infty$, then $\nu$ is quasimonomial on $R$ with $\nu^{\lambda(\nu)-1}=m_{\lambda(\nu)-1}^{\nu} \cdot \operatorname{Ord}_{R_{\lambda(\nu)-1}}$. If $\lambda(\nu)=\infty$, then there exists $i_{0} \geq 1$ such that $R_{j}^{\nu}$ is satellite for $j>i_{0}$ and $R_{i_{0}}^{\nu}$ is free. Note that $R_{i_{0}+1}^{\nu}$ must be proximate to $R_{i_{0}-1}^{\nu}$. By Remark 2, we have $m_{i_{0}-1}^{\nu}>m_{i_{0}}^{\nu}$ and there exists a base $\left(x_{1}^{\left(i_{0}\right)}, x_{2}^{\left(i_{0}\right)}\right)$ of $M\left(R_{i_{0}}^{\nu}\right)$ such that $x_{2}^{\left(i_{0}\right)} R_{i_{0}}^{\nu}=M\left(R_{i_{0}-1}^{\nu}\right) R_{i_{0}}^{\nu}, \nu^{i_{0}}\left(x_{1}^{\left(i_{0}\right)}\right)=m_{i_{0}}^{\nu}$ and $\nu^{i_{0}}\left(x_{2}^{\left(i_{0}\right)}\right)=m_{i_{0}-1}^{\nu}$. By Lemma $5, m_{i_{0}-1}^{\nu}$ and $m_{i_{0}}^{\nu}$ are rationally independent, by Lemma $11, \nu^{i_{0}}$ is monomial on $R_{i_{0}}^{\nu}$ and this complete the proof.

\section{Ordering valuations}

Throughout this section $\nu$ and $\mu$ will be two centered valuations on $R$ such that $0 \leq \mu(f) \leq \nu(f)$ for each $f \in R$. We denote by $\left\{\left(R_{i}^{\nu}, \nu^{i}\right)\right\}_{i=0}^{\lambda(\nu)}$ and $\left\{\left(R_{i}^{\mu}, \mu^{i}\right)\right\}_{i=0}^{\lambda(\mu)}$ the sequences of quadratic dilatations of $R$ along $\nu$ and $\mu$, respectively; and by $\left\{m_{i}^{\nu}\right\}_{i=0}^{\lambda(\nu)}$ and $\left\{m_{i}^{\mu}\right\}_{i=0}^{\lambda(\mu)}$ the multiplicity sequences attached to the pairs $(R, \nu)$ and $(R, \mu)$, respectively.

The objective of this section is to study the relation between the above sequences.

Lemma 17. With the above notations, let us assume that $\mu(x)=\nu(x)<\infty$ and $0 \leq \mu(y)<\min \{\nu(y), \mu(x)\}$ for some base $(x, y)$ of $M(R)$, then $\mu$ is a monomial valuation on $R$ with respect to $(x, y)$.

Proof. If $\mu(x)$ and $\mu(y)$ are rationally independent, then the result follows from Lemma 11. Therefore, let us assume that $\mu(x)$ and $\mu(y)$ are rationally dependent and write $p \mu(x)=q \mu(y)$ with $0 \leq p<q$ coprime integers.

If $\mu$ is not a monomial valuation on $R$ with respect to $(x, y)$, there exists $f=$ $\sum_{i \mu(x)+j \mu(y)=\alpha} a_{i j} x^{i} y^{j} \in R-\{0\}$ with $a_{i j}=0$ or $a_{i j} \in R-M(R)$ and such that $\mu(f)>\alpha$. Without loss of generality, we can assume $f \notin x R$. Thus, we can write $f=\sum_{i=0}^{\lambda} a_{\lambda-i}\left(x^{p}\right)^{\lambda-i}\left(y^{q}\right)^{i}$ with $a_{0} \in R-M(R)$ and $\mu(f)>p \lambda \mu(x)=q \lambda \mu(y)=\alpha$.

Hence, $p(\lambda-\beta) \nu(x)+q \beta \nu(y)>p(\lambda-\beta) \mu(x)+q \beta \mu(y)=p \lambda \mu(x)=p \lambda \nu(x)$, for $0<\beta \leq \lambda$ and $\nu(f)=p \lambda \mu(x)=p \lambda \nu(x)<\mu(f)$, which is a contradiction.

Theorem 18. With the above notations, let us assume that there exists $s \geq 0$ such that $\operatorname{dim}\left(R_{i}^{\mu}\right)=2, m_{i}^{\nu}=m_{i}^{\mu}$ for $0 \leq i \leq s$ and either $\operatorname{dim}\left(R_{s+1}^{\nu}\right)=1$ or $\operatorname{dim}\left(R_{s+1}^{\nu}\right)=$ 2 and $m_{s+1}^{\nu}>m_{s+1}^{\mu}$. Then $R_{i}^{\nu}=R_{i}^{\mu}$ and $0 \leq \mu^{i}(f) \leq \nu^{i}(f)$ for each $f \in R_{i}$, $0 \leq i \leq s$. Moreover, $R_{s+1}^{\nu}$ is free and we have the following possibilities: 
a) If $\operatorname{dim}\left(R_{s+1}^{\nu}\right)=1$, then $\lambda(\mu)=\lambda(\nu)=s+1$ and $\mu^{s}=\nu^{s}=m_{s}^{\nu} \cdot \operatorname{Ord}_{R_{s}}$.

b) If $\operatorname{dim}\left(R_{s+1}^{\nu}\right)=2$ and $\operatorname{dim}\left(R_{s+1}^{\mu}\right)=1$, then $s+1=\lambda(\mu)<\lambda(\nu)$ and there exists a monomial valuation $\bar{\mu}^{s+1}$ on $R_{s+1}^{\nu}$ such that $\mu^{s}$ is the restriction of $\bar{\mu}^{s+1}$ to $R_{s}^{\mu}$. In fact, $\mu^{s}=m_{s}^{\mu} \cdot \operatorname{Ord}_{R_{s}^{\mu}}$.

c) If $\operatorname{dim}\left(R_{s+1}^{\nu}\right)=2=\operatorname{dim}\left(R_{s+1}^{\mu}\right)=2$, then $s+1<\min \{\lambda(\mu), \lambda(\nu)\}, R_{s+1}^{\nu}=$ $R_{s+1}^{\mu}, 0 \leq \mu^{s+1}(f) \leq \nu^{s+1}(f)$ for all $f \in R_{s+1}$ and $\mu^{s+1}$ is a monomial valuation on $R_{s+1}^{\mu}=R_{s+1}^{\nu}$.

Proof. Let $\left(x_{1}, x_{2}\right)$ be a base of $M(R)$. Since $s \geq 0$, then $m_{0}^{\nu}=m_{0}^{\mu}$ and we can assume $\nu\left(x_{1}\right)=\mu\left(x_{1}\right)=m_{0}^{\nu}=m_{0}^{\mu} \leq \mu\left(x_{2}\right) \leq \nu\left(x_{2}\right)$. Note that $R=R_{0}^{\mu}=R_{0}^{\nu}$ and $0 \leq \mu(f) \leq \nu(f)$ for all $f \in R$.

If $A=R\left[\frac{x_{2}}{x_{1}}\right]$, then $A \subset R_{1}^{\nu}, A \subset R_{1}^{\mu}, R_{1}^{\mu}=A_{Q}$ and $R_{1}^{\nu}=A_{Q^{\prime}}$, where $Q=\{h \in$ $\left.A ; \mu^{1}(h)>0\right\}$ and $Q^{\prime}=\left\{h \in A ; \nu^{1}(h)>0\right\}$. Moreover, if $h \in Q$, then $\left(x_{1}\right)^{d} h \in R$ for some $d \geq 0$. Hence, $\mu\left(\left(x_{1}\right)^{d} h\right)=\mu^{1}(h)+d \mu\left(x_{1}\right) \leq \nu\left(\left(x_{1}\right)^{d} h\right)=\nu^{1}(h)+d \mu\left(x_{1}\right)$, $\nu^{1}(Q)>0$ and $Q \subset Q^{\prime}$.

Now, we have the following possibilities:

A) $\operatorname{dim}\left(R_{1}^{\mu}\right)=2$. In this case, $Q$ is a maximal ideal of $A$, so $Q=Q^{\prime}$ and $R_{1}^{\mu}=R_{1}^{\nu}$. In particular, $\operatorname{dim}\left(R_{1}^{\nu}\right)=2,0 \leq \mu^{1}(f) \leq \nu^{1}(f)$ for each $f \in R_{1}^{\mu}=R_{1}^{\nu}$ and $R_{1}^{\mu}=R_{1}^{\nu}$ is free. Moreover, if $m_{1}^{\mu}<m_{1}^{\nu}$, then $s=0, s+1=1<\min \{\lambda(\mu), \lambda(\nu)\}$ and we can take a base $\left(x_{1}^{(1)}, x_{2}^{(1)}\right)$ of $M\left(R_{1}^{\nu}\right)=M\left(R_{1}^{\mu}\right)$ such that $x_{2}^{(1)} R_{1}^{\nu}=M\left(R_{0}^{\nu}\right) R_{1}^{\nu}$, $\mu^{1}\left(x_{2}^{(1)}\right)=\nu^{1}\left(x_{2}^{(1)}\right)=m_{0}^{\mu}=m_{0}^{\nu}$ and $m_{1}^{\mu}=\mu^{1}\left(x_{1}^{(1)}\right)<\min \left\{\mu^{1}\left(x_{2}^{(1)}\right), \nu^{1}\left(x_{1}^{(1)}\right)\right\}$. Finally, by Lemma $17, \mu^{1}$ is a monomial valuation on $R_{1}^{\mu}$ with respect to $\left(x_{1}^{(1)}, x_{2}^{(1)}\right)$.

B) $\operatorname{dim}\left(R_{1}^{\nu}\right)=1$. In this case, $x_{1} A \subset Q \subset Q^{\prime}=x_{1} A$. Thus, $\operatorname{dim}\left(R_{1}^{\mu}\right)=1, s=0$ and $\lambda(\mu)=\lambda(\nu)=1=s+1$. Moreover, $\mu=\mu^{0}=\nu=\nu^{0}=m_{0}^{\nu} \cdot \operatorname{Ord}_{R}$ and $R_{1}^{\nu}$ is free.

C) $\operatorname{dim}\left(R_{1}^{\nu}\right)=2$ and $\operatorname{dim}\left(R_{1}^{\mu}\right)=1$. In this case, $s=0, \lambda(\mu)=1=s+1<\lambda(\nu)$. Moreover, $\mu=\mu^{0}=m_{0}^{\mu} \cdot \operatorname{Ord}_{R_{0}^{\mu}}, R_{1}^{\nu}$ is free and $\mu=\mu^{0}$ is the restriction of $\bar{\mu}^{1}$ to $R_{0}^{\mu}$, where $\left(x_{1}^{(1)}, x_{2}^{(1)}\right)$ is a base of $M\left(R_{1}^{\nu}\right)$ such that $x_{1}^{(1)} R_{1} \nu=M\left(R_{0}^{\nu}\right) R_{1}^{\nu}$ and $\bar{\mu}^{1}$ is the (non-centered) monomial valuation on $R_{1}^{\nu}$, given by $\bar{\mu}^{1}\left(x_{1}^{(1)}\right)=m_{0}^{\nu}=m_{0}^{\mu}$ and $\bar{\mu}^{1}\left(x_{2}^{(1)}\right)=0$.

D) The remainder possibility is $\operatorname{dim}\left(R_{1}^{\mu}\right)=\operatorname{dim}\left(R_{1}^{\nu}\right)=2, m_{1}^{\mu}=m_{1}^{\nu}$ and $0 \leq \mu^{1}(f) \leq \nu^{1}(f)$ for each $f \in R_{1}^{\nu}=R_{1}^{\mu}$. At this point, the result follows from an easy induction.

To finish, we need to show that $R_{s+1}^{\nu}$ is free when $s \geq 1$. Otherwise, $R_{s+1}^{\nu}$ is satellite, $\operatorname{dim}\left(R_{s+1}^{\nu}\right)=2$ and there exists $j<s$ such that $R_{s+1}^{\nu}$ is proximate to $R_{j}^{\nu}$ and $m_{j}^{\nu}=m_{j}^{\mu}>m_{j+1}^{\nu}=m_{j+1}^{\mu}$. (See Remark 2). Furthermore, if $\mathcal{P}\left(R_{j}^{\nu}\right)=$ $\left\{R_{j+1}^{\nu}, \ldots R_{j+h(j)}^{\nu}\right\}$, we have $\mathcal{P}\left(R_{j}^{\mu}\right)=\left\{R_{j+1}^{\mu}, \ldots R_{j+h(j)}^{\mu}\right\}$. (See Remark 4). In fact, $m_{j+k}^{\nu}=m_{j+k}^{\mu}$ for $0 \leq k \leq h(i), R_{s+1}^{\mu}$ is satellite, $\operatorname{dim}\left(R_{s+1}^{\mu}\right)=2$ and $m_{s+1}^{\nu}=m_{s+1}^{\mu}$, which is a contradiction.

Remark 19. With the notations of Theorem 18, we note that $\mu$ is a quasimonomial valuation on $R$. If $\operatorname{dim}\left(R_{s+1}^{\nu}\right)=1$, then $\mu^{s}=\nu^{s}=m_{s}^{\nu} \cdot \operatorname{Ord}_{R_{s}}$; and if $\operatorname{dim}\left(R_{s+1}^{\nu}\right)=2$, there exists a monomial valuation $\bar{\mu}^{s+1}$ on $R_{s+1}^{\nu}$ with respect to a base $\left(x_{1}^{(s+1)}, x_{2}^{(s+1)}\right)$ of $M\left(R_{s+1}^{\nu}\right)$ such that $x_{2}^{(s+1)} R_{s+1}^{\nu}=M\left(R_{s}^{\nu}\right) R_{s+1}^{\nu}, \bar{\mu}^{s+1}\left(x_{2}^{(s+1)}\right)=$ 
$m_{s}^{\mu}=m_{s}^{\nu}, \bar{\mu}^{s+1}\left(x_{1}^{(s+1)}\right)=\alpha$ and $\mu^{i}$ is the restriction of $\bar{\mu}^{s+1}$ to $R_{i}^{\mu}, 1 \leq i \leq s$. In fact, if $\operatorname{dim}\left(R_{s+1}^{\mu}\right)=1$, then $\alpha=0$ and $\mu^{s}=m_{s}^{\nu} \cdot \operatorname{Ord}_{R_{s}^{\nu}}$; and if $\operatorname{dim}\left(R_{s+1}^{\mu}\right)=2$, then $\alpha=m_{s+1}^{\mu}$ and $\mu^{s+1}=\bar{\mu}^{s+1}$.

Corollary 20. With the notations of Theorem 18, if $\nu$ is a quasimonomial valuation on $R$, then $m h(\mu) \leq m h(\nu)$.

Proof. By Remark 19, either $\mu^{s}$ is a monomial valuation on $R_{s}^{\nu}=R_{s}^{\mu}$ or $\mu^{s+1}$ is a monomial valuation on $R_{s+1}^{\nu}=R_{s+1}^{\mu}$. Thus, $h m(\mu) \leq s+1$ in any case.

If $m h(\nu)>s$, there is nothing to do. Therefore, let us assume $m h(\nu) \leq s$ and write $r=m h(\nu)$. By Theorem 18, we have $R_{r}^{\mu}=R_{r}^{\nu}, m_{r}^{\mu}=m_{r}^{\nu}$ and $0 \leq \mu^{r}(f) \leq \nu^{r}(f)$ for each $f \in R_{r}^{\mu}=R_{r}^{\nu}$. In particular, we can take a base $\left(x_{1}^{(r)}, x_{2}^{(r)}\right)$ of $M\left(R_{r}^{\mu}\right)=$ $M\left(R_{r}^{\nu}\right)$ such that $\nu^{r}$ is a monomial valuation on $R_{r}^{\mu}=R_{r}^{\nu}$ with respect to $\left(x_{1}^{(r)}, x_{2}^{(r)}\right)$. Furthermore, we can assume $\mu^{r}\left(x_{1}^{(r)}\right)=m_{r}^{\mu}=m_{r}^{\nu}=\nu^{r}\left(x_{1}^{(r)}\right)$. Hence, by Lemma 12, $\mu^{r}$ is a monomial valuation on $R_{r}^{\mu}=R_{r}^{\nu}$ with respect to $\left(x_{1}^{(r)}, x_{2}^{(r)}\right)$ and $m h(\mu) \leq r=$ $m h(\nu)$.

Proposition 21. With the above notations, let us assume $\lambda(\nu)=\lambda(\mu)$ and $m_{i}^{\nu}=m_{i}^{\mu}$ for $0 \leq i \leq \lambda(\nu)=\lambda(\mu)$, then $\mu=\nu$.

Proof. Let us write $M(R)=\left(x_{1}, x_{2}\right)$ with $\mu\left(x_{1}\right)=\nu\left(x_{1}\right) \leq \mu\left(x_{2}\right) \leq \nu\left(x_{2}\right)$. If $A=$ $R\left[\frac{x_{2}}{x_{1}}\right]$, then $A \subset R_{1}^{\nu}$ and $A \subset R_{1}^{\mu}$. Moreover, if $Q=\left\{h \in A ; \mu^{1}(h)>0\right\}$ and $Q^{\prime}=\left\{h \in A ; \nu^{1}(h)>0\right\}$, then $R_{1}^{\mu}=A_{Q}, R_{1}^{\nu}=A_{Q^{\prime}}$ and $Q \subset Q^{\prime}$ (as in the proof of Theorem 18).

Now, if $\lambda(\nu)=\lambda(\mu)=1$, then $\operatorname{dim}\left(R_{1}^{\mu}\right)=\operatorname{dim}\left(R_{1}^{\nu}\right)=1, x_{1} A=Q=Q^{\prime}$ and $R_{1}^{\nu}=R_{1}^{\mu}=A_{x_{1} A}$. Hence, $\mu=\nu=m_{0}^{\mu} \cdot \operatorname{Ord}_{R}=m_{0}^{\nu} \cdot \operatorname{Ord}_{R}$.

If $\lambda(\nu)=\lambda(\mu)>1$, then $\operatorname{dim}\left(R_{1}^{\mu}\right)=2, Q$ is a maximal ideal of $A, Q=Q^{\prime}$ and $R_{1}^{\mu}=A_{Q}=R_{1}^{\nu}$. Therefore, we can apply induction to get $R_{i}^{\mu}=R_{i}^{\nu}$ for $0 \leq i \leq$ $\lambda(\nu)=\lambda(\mu)$.

On the other hand, from Lemma 1, we get $\mu(g)=\nu(g)$, for each $g \in R$.

\section{The valuative tree}

Next, we prove our main result concerning the tree structure of centered valuations on $R$.

First, hereinafter for each centered valuation $\omega$ on $R$ we denote by $\left\{\left(R_{i}^{\omega}, \omega^{i}\right)\right\}_{i=0}^{\lambda(\omega)}$ (resp. by $\left.\left\{m_{i}^{\omega}\right\}_{i=0}^{\lambda(\omega)}\right)$ the sequence of quadratic dilatations of $R$ along $\omega$ (resp. the multiplicity sequence attached to $(R, \omega))$. Furthermore, we write $\mathcal{F}(\omega)=\left\{i ; R_{i}^{\omega}\right.$ is free $\}$. Thus in what follows, one must replace $\omega$ by the appropriate valuation $\nu, \mu, \mu_{j}, \ldots$, to obtain the notation for each case.

Two valuations $\nu$ and $\mu$ on $R$ are equivalent $(\nu \sim \mu)$ if there exists a non-negative real number $C>0$ such that $\mu(f)=C \cdot \nu(f)$ for all $f \in R$. Here $C \cdot \infty=\infty$. Note that if $\nu \sim \mu$, then $\lambda(\nu)=\lambda(\mu), R_{i}^{\nu}=R_{i}^{\mu}$ and $\nu^{i} \sim \mu^{i}, 0 \leq i \leq \lambda(\nu)=\lambda(\mu)$. In particular, $\bigcup_{i=0}^{\lambda(\nu)} R_{i}^{\nu}=\bigcup_{i=0}^{\lambda(\mu)} R_{i}^{\mu}$ and the Krull valuations that define $\nu$ and $\mu$ are equivalent (see section 2 and [B], p. 99). 
We pick only one element in each equivalence class and consider normalized valuations, i.e., centered valuations $\nu$ on $R$ with $\nu(M(R))=\min \{\nu(y) ; y \in M(R)\}=1$. Note that $\nu(M(R))=\min \left\{\nu\left(x_{1}\right), \nu\left(x_{2}\right)\right\}$, where $M(R)=\left(x_{1}, x_{2}\right)$.

We denote by $\mathcal{V}$ the set of normalized centered valuations on $R$ and by $\leq_{R}$ the natural partial order in $\mathcal{V}$ given by $\mu \leq_{R} \nu$ iff $\mu(f) \leq \nu(f)$ for all $f \in R, \mu, \nu \in \mathcal{V}$. Note that $\operatorname{Ord}_{R} \in \mathcal{V}$ and $\operatorname{Ord}_{R} \leq_{R} \nu$ for all $\nu \in \mathcal{V}$.

Notice that if $R$ is a complete or henselian ring, then the elements of $\mathcal{V}$ can be identified with the real rank one valuation rings that birationally contains $R$. (See section 2$)$.

Let $(\mathcal{T}, \leq)$ be a partially ordered set, or poset, we say that a totally ordered subset $S \subset \mathcal{T}$ is full if $\sigma, \sigma^{\prime} \in S, \tau \in \mathcal{T}$ and $\sigma \leq \tau \leq \sigma^{\prime}$ imply $\tau \in S$.

Definition 22. A rooted non-metric tree is a poset $(\mathcal{T}, \leq)$ such that

(T1) $\mathcal{T}$ has a unique minimal element $\tau_{0}$, called the root of $\mathcal{T}$;

(T2) if $\tau \in \mathcal{T}$, then the set $\{\sigma \in \mathcal{T} ; \sigma \leq \tau\}$ is isomorphic to a real interval;

(T3) every full, totally ordered subset of $\mathcal{T}$ is isomorphic to a real interval.

We point out that if (T1) and (T2) hold, then (T3) is equivalent to

(T3') If $S$ is a totally ordered subset of $\mathcal{T}$ without upper bound in $\mathcal{T}$, then there exists a countable increasing sequence in $S$ without upper bound in $\mathcal{T}$. (See Remark 3.3 of [FJ1]).

Finally, a rooted non-metric tree $\mathcal{T}$ is complete if every increasing sequence $\left(\tau_{i}\right)_{i \geq 1}$ in $\mathcal{T}$ has a majorant, i.e. an element $\tau_{\infty} \in \mathcal{T}$ with $\tau_{i} \leq \tau_{\infty}$ for every $i$.

In what follows, we consider the map $\mathcal{G}: \mathcal{V} \longrightarrow[1, \infty]$ given by $\mathcal{G}(\nu)=\sum_{i \in \mathcal{F}(\nu)} m_{i}^{\nu}$. Here $\mathcal{G}(\nu)=\infty$ when $\lambda(\nu)=\infty$ and the series $\sum_{i \in \mathcal{F}(\nu)} m_{i}^{\nu}$ diverges.

The next results state the main properties of $\mathcal{G}$.

Lemma 23. With the above notations, $\mathcal{G}$ is a strictly increasing map.

Proof. Let $\mu, \nu \in \mathcal{V}$ be such that $\mu<_{R} \nu$.

As $m_{o}^{\nu}=m_{0}^{\mu}=1$ and $R=R_{0}^{\nu}=R_{0}^{\mu}$, then, by Theorem 18 and Proposition 21 , there exists $k \in \mathcal{F}(\nu)$ with $\operatorname{dim}\left(R_{i}^{\mu}\right)=2, m_{i}^{\mu}=m_{i}^{\nu}$ for $0 \leq i \leq k-1$ and either $\operatorname{dim}\left(R_{k}^{\mu}\right)=1$ or $\operatorname{dim}\left(R_{k}^{\mu}\right)=2$ and $m_{k}^{\mu}<m_{k}^{\nu}$. Furthermore, $R_{i}^{\mu}=R_{i}^{\nu}$ and $0 \leq \mu^{i}(f) \leq \nu^{i}(f)$ for each $f \in R_{i}^{\mu}=R_{i}^{\nu}, 0 \leq i \leq k-1$.

If $\operatorname{dim}\left(R_{k}^{\nu}\right)=1$, then $\mu^{k-1}=\nu^{k-1}=m_{k-1}^{\nu} \cdot \operatorname{Ord}_{R_{k-1}}$ and $\mu=\nu$, which is a contradiction, see Theorem 18 and Remark 19. Therefore, $\operatorname{dim}\left(R_{k}^{\nu}\right)=2$.

By Remark 19, there exists a monomial valuation $\bar{\mu}^{k}$ of $R_{k}^{\nu}$ with respect to a base $\left(x_{1}^{(k)}, x_{2}^{(k)}\right)$ of $M\left(R_{k}^{\nu}\right)$ such that $x_{2}^{(k)} R_{k}^{\nu}=M\left(R_{k-1}^{\nu}\right) R_{k}^{\nu}, \bar{\mu}^{k}\left(x_{2}^{(k)}\right)=m_{k-1}^{\mu}=m_{k-1}^{\nu}$, $\bar{\mu}^{k}\left(x_{1}^{(k)}\right)=\alpha$ and $\mu^{i}$ is the restriction of $\bar{\mu}^{k}$ to $R_{i}^{\mu}, 1 \leq i \leq k-1$. Moreover, if $\operatorname{dim}\left(R_{k}^{\mu}\right)=1$, then $\alpha=0$ and $\mu^{k-1}=m_{k-1}^{\nu} \cdot \operatorname{Ord}_{R_{k-1}^{\nu}} ;$ and if $\operatorname{dim}\left(R_{k}^{\mu}\right)=2$, then $\alpha=m_{k}^{\mu}$ and $\mu^{k}=\bar{\mu}^{k}$.

Now, if $\alpha \neq 0$, then $m_{k-1}^{\nu}=m_{k-1}^{\mu} \geq m_{k-1}^{\nu}>m_{k-1}^{\mu}=\alpha$ and $R_{k+j}^{\mu}$ is proximate to $R_{k-1}^{\mu}=R_{k-1}^{\nu}$ for $1 \leq j \leq h_{1}$, where $m_{k-1}^{\nu}=h_{1} \alpha+\alpha_{2}$ with $0 \leq \alpha_{2}<\alpha$. Hence, by Lemma 5, we have:

a) $\mathcal{F}(\mu)=\left\{l_{0}=0<l_{1}=1<l_{2}<\cdots<l_{s}=k\right\}$, when either $\alpha=0$ or $\alpha \neq 0$ and $\alpha$ and $m_{k-1}^{\nu}=m_{k-1}^{\mu}$ are rationally independent. 
b) $\mathcal{F}(\mu)=\left\{l_{0}=0<l_{1}=1<l_{2}<\cdots<l_{s}=k<l_{s+1}\right\}$, when $\alpha \neq 0$ and $\alpha$ and $m_{k-1}^{\nu}=m_{k-1}^{\mu}$ are rationally dependent. In this case, $\operatorname{dim}\left(R_{l_{s+1}}^{\mu}\right)=1$ and $m_{l_{s+1}}^{\mu}=0$.

Hence, $\mathcal{G}(\mu)=\sum_{i \in \mathcal{F}(\mu)} m_{i}^{\mu}=\sum_{j=0}^{s} m_{l_{j}}^{\mu}=\sum_{j=0}^{s-1} m_{l_{j}}^{\nu}+m_{l_{s}}^{\mu}<\sum_{j=0}^{s} m_{l_{j}}^{\nu} \leq \mathcal{G}(\nu)$ in both of cases.

Lemma 24. With the above notations, let us consider $\nu \in \mathcal{V}$, then the set $I=\{\mu \in$ $\left.\mathcal{V} ; \mu \leq_{R} \nu\right\}$ is a full, totally ordered subset of $\mathcal{V}$.

Proof. We only need to see that $I$ is a totally ordered set.

Let us consider $\mu_{1}, \mu_{2} \in I$. If $\mu_{1}=\nu$ or $\mu_{2}=\nu$, there is nothing to do. Thus, we can assume $\mu_{j}<_{R} \nu, j=1,2$ and $\mu_{1} \neq \mu_{2}$.

By Theorem 18, for $j=1,2$ there exists $k_{j} \in \mathcal{F}(\nu)$ such that $\operatorname{dim}\left(R_{i}^{\mu_{j}}\right)=2$, $m_{i}^{\mu_{j}}=m_{i}^{\nu}$ for $0 \leq i \leq k_{j}-1$ and either $\operatorname{dim}\left(R_{l_{k_{j}}}^{\mu_{j}}\right)=1$ or $\operatorname{dim}\left(R_{k_{j}}^{\mu_{j}}\right)=2$ and $m_{k_{j}}^{\mu_{j}}<m_{k_{j}}^{\nu}$. Furthermore, $R_{i}^{\mu_{j}}=R_{i}^{\nu}$ and $0 \leq \mu_{j}^{i}(f) \leq \nu^{i}(f)$ for each $f \in R_{i}^{\mu_{j}}=R_{i}^{\nu}$, $0 \leq i \leq k_{j}-1,1 \leq j \leq 2$.

Since $\mu_{j}<_{R} \nu$, then $\operatorname{dim}\left(R_{k_{j}}^{\nu}\right)=2,1 \leq j \leq 2$. (Otherwise, $\mu_{j}=\nu$ by Remark 19). Thus (and also by Remark 19), there exists a monomial valuation $\bar{\mu}_{j}^{k_{j}}$ on $R_{k_{j}}^{\nu}$ with respect to a base $\left(x_{1}^{\left(k_{j}\right)}, x_{2}^{\left(k_{j}\right)}\right)$ of $M\left(R_{k_{j}}^{\nu}\right)$ such that $x_{2}^{\left(k_{j}\right)} R_{k_{j}}^{\nu}=M\left(R_{k_{j}-1}^{\nu}\right) R_{k_{j}}^{\nu}$, $\bar{\mu}_{j}^{k_{j}}\left(x_{2}^{\left(k_{j}\right)}\right)=m_{k_{j}-1}^{\mu_{j}}=m_{k_{j}-1}^{\nu}, \bar{\mu}_{j}^{k_{j}}\left(x_{1}^{\left(k_{j}\right)}\right)=\alpha_{j}$ and $\mu_{j}^{i}$ is the restriction of $\bar{\mu}_{j}^{k_{j}}$ to $R_{i}^{\mu_{j}}$ $1 \leq i \leq k_{j}-1$. Moreover, if $\operatorname{dim}\left(R_{k_{j}}^{\mu_{j}}\right)=1$, then $\alpha_{j}=0$ and $\mu_{j}^{k_{j}-1}=m_{k_{j}-1}^{\nu} \operatorname{Ord}_{R_{k_{j}-1}^{\nu}}$; and if $\operatorname{dim}\left(R_{k_{j}}^{\mu_{j}}\right)=2$, then $\alpha_{j}=m_{k_{j}}^{\mu_{j}}$ and $\mu_{j}^{k_{j}}=\bar{\mu}_{j}^{k_{j}}$. Therefore, $\alpha_{j}<m_{k_{j}}^{\nu}, j=1,2$.

Without loss of generality, we can assume $k_{1} \leq k_{2}$. If $k_{1}<k_{2}$, then $\bar{\mu}_{1}^{k_{1}} \leq_{R_{k_{1}}^{\nu}} \mu_{2}^{k_{1}}$ and $\mu_{1} \leq_{R} \mu_{2}$; and if $k_{1}=k_{2}$, then either $\bar{\mu}_{1}^{k_{1}} \leq_{R_{k_{1}}^{\nu}} \bar{\mu}_{2}^{k_{1}}$ and $\mu_{1} \leq_{R} \mu_{2}$ when $\alpha_{1} \leq \alpha_{2}$ or $\bar{\mu}_{2}^{k_{1}} \leq_{R_{k_{1}}^{\nu}} \bar{\mu}_{1}^{k_{1}}$ and $\mu_{2} \leq_{R} \mu_{1}$ when $\alpha_{2} \leq \alpha_{1}$. Note that if $k_{1}=k_{2}$, we can take $x_{i}^{\left(k_{1}\right)}=x_{i}^{\left(k_{2}\right)}, i=1,2$.

Lemma 25. With the above notations, let us consider $\nu \in \mathcal{V}$ and $I=\left\{\mu \in \mathcal{V} ; \mu \leq_{R}\right.$ $\nu\}$, then the restriction $\mathcal{G}_{I}$ of $\mathcal{G}$ to $I$ is a strictly increasing one-to-one map from $I$ onto $[1, \mathcal{G}(\nu)]$.

Proof. By Lemmas 23 and 24, $\mathcal{G}_{I}$ is a strictly increasing one-to-one map from $I$ on $[1, \mathcal{G}(\nu)]$. Therefore, we only need to see that $\mathcal{G}_{I}$ is an onto map.

Let us consider $\beta \in[1, \mathcal{G}(\nu)]$, then there exits $k \in \mathcal{F}(\nu)$ such that

$$
\sum_{j \in \mathcal{F}(\nu), j<k} m_{j}^{\nu} \leq \beta<\sum_{j \in \mathcal{F}(\nu), j \leq k} m_{j}^{\nu} .
$$

We write $\alpha=\beta-\sum_{j \in \mathcal{F}(\nu), j<k} m_{j}^{\nu}$. Note that $\alpha<m_{k}^{\nu}$.

As $k$ is a majorant of $\mathcal{F}(\nu)$, if $\operatorname{dim}\left(R_{k}^{\nu}\right)=1$, then $m_{k}^{\nu}=0$ and $\beta=\mathcal{G}(\nu)$.

If $\operatorname{dim}\left(R_{k}^{\nu}\right)=2$, let ${ }^{\alpha} \mu^{k}$ be the monomial valuation on $R_{k}^{\nu}$ with respect to a base $\left(x_{1}^{(k)}, x_{2}^{(k)}\right)$ of $M\left(R_{k}\right)$ with $x_{2}^{(k)} R_{k}=M\left(R_{k-1}\right) R_{k},{ }^{\alpha} \mu^{k}\left(x_{1}^{(k)}\right)=\alpha$ and ${ }^{\alpha} \mu^{k}\left(x_{2}^{(k)}\right)=$ $m_{k-1}^{\nu}>0$. Note that if $\alpha>0$, then ${ }^{\alpha} \mu^{k}$ is a centered valuation on $R_{k}^{\nu}$ and, in general, ${ }^{\alpha} \mu^{k}\left(R_{k}^{\nu}\right) \geq 0$. 
For $0 \leq j \leq k-1$, let ${ }^{\alpha} \mu^{j}$ be the restriction of ${ }^{\alpha} \mu^{k}$ to $R_{j}^{\nu}$, then ${ }^{\alpha} \mu^{j}$ is a centered valuation on $R_{j}^{\nu}$ for $0 \leq j \leq k-1$ and ${ }^{\alpha} \mu^{j} \leq_{R_{j}^{\nu}} \nu^{j}$ for $0 \leq j \leq k$.

To simplify notation, we write $\mu={ }^{\alpha} \mu^{0}$. We have $R_{i}^{\mu}=R_{i}^{\nu}$ and $\mu^{i}={ }^{\alpha} \mu^{i}$ for $0 \leq i \leq k-1$.

Next, we will show by induction that $m_{j}^{\nu}=m_{j}^{\mu}$ for $0 \leq j \leq k-1$.

First, since ${ }^{\alpha} \mu^{k}\left(R_{k}^{\nu}\right) \geq 0$, we have $m_{k-1}^{\mu}=m_{k-1}^{\nu}$.

Now, let us assume $m_{k-i}^{\mu}=m_{k-i}^{\nu}$ for $1 \leq i \leq j$.

If $m_{k-j-1}^{\nu}=m_{k-j}^{\nu}$, then $m_{k-j-1}^{\mu}=m_{k-j}^{\mu}$ and $m_{k-j-1}^{\nu}=m_{k-j-1}^{\mu}$. Thus, let us assume $m_{k-j-1}^{\nu}>m_{k-j}^{\nu}$.

By Remark 2, $R_{k-j+1}^{\nu}$ is proximate to $R_{k-j-1}^{\nu}$ and $m_{k-j-1}^{\mu}>m_{k-j}^{\mu}$. Let us write $\mathcal{P}\left(R_{k-j-1}^{\nu}\right)=\left\{R_{k-j-1+l}^{\nu} ; 0 \leq l \leq h\right\}$, since $R_{k}^{\nu}$ is free, then $k+j-1+h<k$ or equivalently $h<j+1$. Also, let us write $\mathcal{P}\left(R_{k-j-1}^{\mu}\right)=\left\{R_{k-j-1+e}^{\mu} ; 0 \leq e \leq h^{\prime}\right\}$. Note that $h^{\prime} \geq h$ and $m_{k-j-1}^{\nu}=\sum_{i=1}^{h} m_{k-j-1+i}^{\nu}=\sum_{i=1}^{h} m_{k-j-1+i}^{\mu} \leq m_{k-j-1}^{\mu} \leq m_{k-j-1}^{\nu}$. Hence, $h^{\prime}=h$ and $m_{k-j-1}^{\nu}=m_{k-j-1}^{\mu}$.

At this point, if $\alpha \neq 0$, we have $m_{k-1}^{\nu}=m_{k-1}^{\mu} \geq m_{k}^{\nu}>\alpha=m_{k}^{\mu}$. Furthermore, if $m_{k-1}^{\nu}=h_{1} \alpha+\alpha_{2}$ with $0 \leq \alpha_{2}<\alpha$, then $R_{k+j}^{\mu}$ is proximate to $R_{k-1}^{\mu}=R_{k-1}^{\nu}$ for $1 \leq j \leq h_{1}$. Hence, by Lemma 5 , we have two possibilities:

a) $\mathcal{F}(\mu)=\left\{l_{0}=0<l_{1}=1<l_{2}<\cdots<l_{s}=k\right\}$ when either $\alpha=0$ or $\alpha \neq 0$ and $\alpha$ and $m_{k-1}^{\nu}$ are rationally independent.

b) $\mathcal{F}(\mu)=\left\{l_{0}=0<l_{1}=1<l_{2}<\cdots<l_{s}=k<l_{s+1}\right\}$, when $\alpha \neq 0$ and $\alpha$ and $m_{k-1}^{\nu}$ are rationally dependent. In this case, $\operatorname{dim}\left(R_{l_{s+1}}^{\mu}\right)=1$ and $m_{l_{s+1}}^{\mu}=0$.

Therefore, $\mathcal{G}(\mu)=\sum_{j=0}^{s-1} m_{l_{j}}^{\mu}+\alpha=\sum_{j=0}^{s-1} m_{l_{j}}^{\nu}+\alpha=\beta$ in any case.

Lemma 26. With the above notations, every totally ordered subset $\mathcal{S} \subset \mathcal{V}$ has a majorant in $\mathcal{V}$.

Proof. Let us write $H=\{m h(\mu) ; \mu \in \mathcal{S}\}$.

If $\infty \in H$, let $\mu \in \mathcal{S}$ be such that $m h(\mu)=\infty$. If there exists $\nu \in \mathcal{S}$ with $\mu<_{R} \nu$, then, by Remark 19, $\mu$ is quasimonomial and $m h(\mu)<\infty$, which is a contradiction. Hence, $\mu$ is a majorant of $\mathcal{S}$.

If $\infty \notin H$, we can distinguish two possibilities:

a) $H$ is infinite. In this case, we take $\left\{\mu_{i}\right\}_{i \geq 1} \subset \mathcal{S}$ with $m h\left(\mu_{i}\right)<m h\left(\mu_{i+1}\right), i \geq 1$. Since $\mathcal{S}$ is a totally ordered set, then $\mu_{i}<\mu_{i+1}$, by Corollary 20 .

Let us consider $\nu: R \longrightarrow \overline{\mathbb{R}}_{+}$given by $\nu(g)=\sup \left\{\mu_{i}(g) ; i \geq 1\right\}$ for each $g \in R$. It is easy to check that $\nu \in \mathcal{V}$ with $\mu_{i} \leq_{R} \nu, i \geq 1$. Furthermore, if $\mu \in \mathcal{S}$, then there exists $i$ with $m h(\mu)<m h\left(\mu_{i}\right)$. Hence, $\mu<_{R} \mu_{i} \leq_{R} \nu$ by Corollary 20, and $\nu$ is a majorant of $\mathcal{S}$.

b) $H$ is finite. In this case, we write $h=\max (H)$ and distinguish two further possibilities:

b.1) There are a finite number of valuations $\mu \in \mathcal{S}$ (say $\mu_{1}, \ldots, \mu_{s}$ ) with $m h(\mu)=h$. Since $\mathcal{S}$ is totally ordered, we can assume $\mu_{i} \leq_{R} \mu_{s}, 1 \leq i \leq s$.

Let us consider $\mu \in \mathcal{S}$. If $m h(\mu)<m h\left(\mu_{s}\right)=h$, then $\mu<_{R} \mu_{s}$ by Corollary 20; and if $m h(\mu)=m h\left(\mu_{s}\right)=h$, then $\mu \leq_{R} \mu_{s}$. Hence, $\mu_{s}$ is a majorant of $\mathcal{S}$.

b.2) There are infinitely many valuations $\mu \in \mathcal{S}$ with $m h(\mu)=h$.

By Theorem 18, if $\mu, \nu \in \mathcal{S}$ with $\mu \leq_{R} \nu$ and $m h(\mu)=m h(\nu)=h$, then $m_{i}^{\mu}=m_{i}^{\nu}$, $0 \leq i \leq h-1$ and $m_{h}^{\mu} \leq m_{h}^{\nu}$. 
We denote by $M=\left\{m_{h}^{\mu} ; m h(\mu)=h, \mu \in \mathcal{S}\right\}$ and by $a=\sup (M)$ (note that $m_{h-1}^{\mu}$ is a majorant of $M$ for each $\mu \in \mathcal{S}$ with $\left.m h(\mu)=h\right)$. We have two possibilities:

b.2.i) $a \in M$. In this case, we write $S_{a}=\left\{\mu \in \mathcal{S} ; m h(\mu)=h, m_{h}^{\mu}=a\right\}$ and distinguish two further cases:

b.2.i. $\alpha) S_{a}$ is finite, say $S_{a}=\left\{\mu_{1}<_{R} \mu_{2}<_{R} \cdots<_{R} \mu_{s}\right\}$. As in case b.1), we easily get that $\mu_{s}$ is a majorant of $\mathcal{S}$.

b.2.i. $\beta) S_{a}$ is not finite. In this case, if $\nu, \mu \in S_{a}$, we have $m_{h}^{\mu}=m_{h}^{\nu}=a$ and $R_{i}^{\mu}=R_{i}^{\nu}$ for $0 \leq i \leq h$, by Theorem 18. Furthermore, if $\mu \in S_{a}$, then $\mu^{h}$ is a monomial valuation on $R_{h}^{\mu}$ with respect to a base $\left(x_{1}^{(h)}, x_{2}^{(h)}\right)$ of $M\left(R_{h}^{\mu}\right)$ with $\mu^{h}\left(x_{1}^{(h)}\right) \leq \mu^{h}\left(x_{2}^{(h)}\right)$. In particular, $\mu^{h}\left(x_{1}^{(h)}\right)=m_{h}^{\mu}=a$ and as $\mu^{h}$ is monomial on $R_{h}^{\mu}$, then $\mu^{h}\left(x_{2}^{(h)}\right)=\Omega_{h}^{\mu}=$ $\max \left\{\mu^{h}(y) ; y \in M\left(R_{h}^{\mu}\right)-\left(M\left(R_{h}^{\mu}\right)\right)^{2}\right\}$.

We point out that if $\mu, \nu \in S_{a}$, then $\mu^{h}$ and $\nu^{h}$ are monomial valuations on $R_{h}^{\mu}=R_{h}^{\nu}$ with respect to the same base $\left(x_{1}^{(h)}, x_{2}^{(h)}\right)$ of $M\left(R_{h}^{\nu}\right)$. (Note that $\mu \leq_{R} \nu$ or $\nu \leq_{R} \mu$ and as $m_{h}^{\mu}=m_{h}^{\nu}=a$, we can apply Lemma 12). In particular, $\Omega_{h}^{\mu}<\Omega_{h}^{\nu}$ if and only if $\mu<_{R} \nu$; and $\left\{\Omega_{h}^{\mu} ; \mu \in S_{a}\right\}$ is an infinite set.

Let $\left\{\mu_{i}\right\}_{i \geq 1} \subset S_{a}$ be such that $\Omega_{h}^{\mu_{i}}<\Omega_{h}^{\mu_{i+1}}$. Note that $\mu_{i}<_{R} \mu_{i+1}$ for $i \geq 1$.

Now, let $\nu: R \longrightarrow \overline{\mathbb{R}}_{+}$be given by $\nu(g)=\sup \left\{\mu_{i}(g) ; i \geq 1\right\}$ for each $g \in R$. As in case a), we easily get that $\nu \in \mathcal{V}$ and that $\nu$ is a majorant of $\mathcal{S}$.

b.2.ii) $a \notin M$. In this case, let $\left\{\mu_{i}\right\}_{i \geq 1} \subset \mathcal{S}$ be such that $m h\left(\mu_{i}\right)=h, m_{h}^{\mu_{i}}<m_{h}^{\mu_{i+1}}$ for $i \geq 1$ and $a=\sup \left\{m_{h}^{\mu_{i}} ; i \geq 1\right\}$. Note that $\mu_{i}<\mu_{i+1}$ for $i \geq 1$.

Finally, let $\nu: R \longrightarrow \overline{\mathbb{R}}_{+}$be given by $\nu(g)=\sup \left\{\mu_{i}(g) ; i \geq 0\right\}$ for each $g \in R$. As above $\nu \in \mathcal{V}$ and $\nu$ is a majorant of $\mathcal{S}$.

Corollary 27. With the above notations, let $\mathcal{S}$ be a full, totally ordered subset of $\mathcal{V}$, then $\mathcal{G}(\mathcal{S})$ is order isomorphic to a real interval.

Proof. By Lemma 26, $\mathcal{S}$ has a majorant $\nu \in \mathcal{V}$. Thus, $\mathcal{S} \subset\left[\operatorname{Ord}_{R}, \nu\right]$ and $\mathcal{G}:$ $\left[\operatorname{Ord}_{R}, \nu\right] \longrightarrow[1, \mathcal{G}(\nu)]$ is a strictly increasing order isomorphism. Therefore, $\mathcal{S}$ is order isomorphic to $\mathcal{G}(\mathcal{S})$, which is a full, totally ordered subset of $\mathbb{R}$. Hence, $\mathcal{G}(\mathcal{S})$ is a real interval.

Definition 28. A parameterization of a rooted, non-metric tree $(\mathcal{T}, \leq)$ is an increasing (or decreasing) mapping $\alpha: \mathcal{T} \longrightarrow[-\infty,+\infty]$ whose restriction to any full, totally ordered subset of $\mathcal{T}$ gives a bijection onto a real interval. (See [FJ1], p. 47).

The main result of this paper is the following:

Theorem 29. With the above notations, the valuation space $\left(\mathcal{V}, \leq_{R}\right)$ is a parametrized complete non-metric tree, rooted at $\operatorname{Ord}_{R}$.

Proof. Obviously, $\operatorname{Ord}_{R} \leq_{R} \nu$ for every $\nu \in \mathcal{V}$ and we have (T1). (T2) is consequence of Lemma 25. (T3') and the completion of $\mathcal{V}$ follows from Lemma 26. Thus $\left(\mathcal{V}, \leq_{R}\right)$ is a complete non-metric tree rooted at $\operatorname{Ord}_{R}$. Finally, from Lemma 23 and Corollary $27, \mathcal{G}$ is a parameterization of $\left(\mathcal{V}, \leq_{R}\right)$.

Remark 30. In [FJ1], (3.3.1), the parameterization of $\left(\mathcal{V}, \leq_{R}\right)$ is given by the skewness of $\nu \in \mathcal{V}$, when $R=\mathbb{C}[[X, Y]]$ is the formal power series ring in two variables over the complex field $\mathbb{C}$. Namely, the skewness of $\nu$ is defined by $\alpha(\nu)=$ 
$\sup \left\{\frac{\nu(\phi)}{\operatorname{Ord}_{R}(\phi)} ; \phi \in M(R)-\{0\}\right\}$. In this case, one can see that $\alpha(\nu) \leq \mathcal{G}(\nu)$ for every $\nu \in \mathcal{V}$.

\section{References}

[Ab] S. S. Abhyankar, On the valuations centered in a local domain, Amer. J. Math. 78 (1956), 321-348.

[B] N. Bourbaki, Algèbre Commutative, Chapitres 5 a 7 . (Masson, Paris. 1985).

[FJ1] C. Favre and M. Jonsson, The Valuative Tree, Lecture Notes in Mathematics, 1853, (SpringerVerlag. 2004).

[FJ2] _ Valuative analysis of planar plurisubharmonic functions, Invent. Math. 162 (2005), no. 2, 271-311.

[FJ3] _ Valuations and multiplier ideals, J. Amer. Math. Soc. 18 (2005), no. 4, 655-684.

[Fu] D. Fu, Local weak simultaneous resolution for high rational ranks, J. Algebra 194 (1997), no. 2, 614-630.

[GR] A. Granja and C. Rodríguez, Proximity relations for real rank one valuations dominating a local regular ring, Rev. Mat. Iberoamericana 19 (2003), no. 2, 393-412.

[L] J. Lipman, Proximity inequalities for complete ideals in two-dimensional regular local rings, Contemp. Math. 159, Amer. Math. Soc., Providence, RI, (1994) 293-306.

[M1] S. MacLane, A construction for absolute values in polynomial rings, Trans. Amer. Math. Soc. 40 (1936), no. 3, 363-395.

[M2] - A construction for prime ideals as absolute values of an algebraic field, Duke Math. J. 2 (1936), no. 3, 492-510.

[ZS] O. Zariski and P. Samuel, Commutative Algebra. Vol. I and II, D. Van Nostrand Co., Inc., Princeton, N.J.-Toronto-London-New York, (1960).

Dpto. de Matemáticas, Universidad de León, 24071-León, Spain

E-mail address: demagb@unileon.es 